\title{
Intersections of lung progenitor cells, lung disease and lung cancer
}

\author{
Carla F. Kim ${ }^{1,2,3}$
}

Affiliations: ${ }^{1}$ Stem Cell Program, Division of Hematology/Oncology and Division of Respiratory Disease, Boston Children's Hospital, Boston, MA, USA. ${ }^{2}$ Dept of Genetics, Harvard Medical School, Boston, MA, USA. ${ }^{3}$ Harvard Stem Cell Institute, Cambridge, MA, USA.

Correspondence: Carla F. Kim, Boston Children's Hospital, 300 Longwood Avenue, Boston, MA 02115, USA. E-mail: carla.kimachildrens.harvard.edu

@ERSpublications

Stem cell biology has brought new techniques to the lung field and has elucidated possible therapeutic pathways http://ow.ly/h74x30cA6Lo

Cite this article as: Kim CF. Intersections of lung progenitor cells, lung disease and lung cancer. Eur Respir Rev 2017; 26: 170054 [https://doi.org/10.1183/16000617.0054-2017].

ABSTRACT The use of stem cell biology approaches to study adult lung progenitor cells and lung cancer has brought a variety of new techniques to the field of lung biology and has elucidated new pathways that may be therapeutic targets in lung cancer. Recent results have begun to identify the ways in which different cell populations interact to regulate progenitor activity, and this has implications for the interventions that are possible in cancer and in a variety of lung diseases. Today's better understanding of the mechanisms that regulate lung progenitor cell self-renewal and differentiation, including understanding how multiple epigenetic factors affect lung injury repair, holds the promise for future better treatments for lung cancer and for optimising the response to therapy in lung cancer. Working between platforms in sophisticated organoid culture techniques, genetically engineered mouse models of injury and cancer, and human cell lines and specimens, lung progenitor cell studies can begin with basic biology, progress to translational research and finally lead to the beginnings of clinical trials.

\section{Introduction}

The field of lung stem cell biology has advanced significantly over the past 10 years, beginning with very limited knowledge of how to identify the epithelial cells in lung tissue with stem cell properties and lacking functional, physiological assays to determine if lung epithelial cells have the ability to self-renew or give rise to differentiated cell types. Without these tools, it was not possible to define the pathways that regulate progenitor cell activity in the lung. At that time, it was also unclear whether cells that sustained the largely quiescent lung epithelium could be activated to give rise to different lineages after injury. Cell-sorting strategies adopted from protocols used to isolate lung epithelial cells, combined with the use of markers used in the haematopoietic system, made it possible to enrich for lung cells with the ability to self-renew and to give rise to bronchiolar and alveolar cell types in culture [1,2]. A variety of techniques have been used to prepare lung tissue for cell sorting and other types of single-cell analysis, used in

This article has been revised according to the correction published in the December 2017 issue of the European Respiratory Review.

Received: May 082017 | Accepted after revision: May 192017

Support statement: Funding was received from the National Institutes of Health (US Dept of Health and Human Services, Bethesda, MD, USA), grant number RO1 HL090136. Funding information for this article has been deposited with the Crossref Funder Registry.

Conflict of interest: None declared.

Provenance: Commissioned article, peer reviewed.

Copyright OERS 2017. ERR articles are open access and distributed under the terms of the Creative Commons Attribution Non-Commercial Licence 4.0. 
combination with various approaches using cell surface antibodies or other reporter alleles to identify and enrich for putative progenitor cells [3-7].

\section{Culture techniques}

Recent developments in organoid co-culture studies have been transformative in providing functional assays for lung progenitor cell activity that allow analysis of cell-cell interactions in differentiation and disease. For proximal lung progenitor cells, such as basal cells, air-liquid interface culture systems have long offered a fruitful way to assess progenitor cell activity. Even prior to the use of fluorescence-activated cell sorting with cell surface markers to enrich for basal cells, preparations of tracheal epithelial cells from mouse and human have been used to yield differentiated cultures containing ciliated cells and other more differentiated cell types [8]. While the air-liquid interface method has been a seminal source of understanding differentiation, it has not been used to dissect how different cell types, such as stromal cells, affect this process. Culturing alveolar epithelial cells on collagen or Matrigel, an extracellular protein mixture used frequently as a substrate for cells in culture and in transplantations, has been useful for assessing alveolar type II cell differentiation to alveolar type I cells $[9,10]$. One limitation of alveolar type II cell culture techniques has been the inability to maintain the cells in a proliferative state over multiple passages without substantial differentiation or morphological changes. Organoid culture systems, i.e. primary cell cultures in which individual or multiple epithelial progenitor cells proliferate and self-organise into structures that resemble the cellular arrangement in the tissue, provide a new tool to understand epithelial biology. The term "organoid" was perhaps originally used to refer to cultures of pieces of tissue maintained in culture; tissue bits were kept intact so that connective tissue, epithelia and stroma were represented in the culture [11]. Today's organoid co-cultures may perhaps be thought of as organotypic in comparison: by mixing various cell types such as epithelial cells and stroma with a substrate of choice, we can reconstruct a tissue of interest with organoids. This strategy, in comparison to previously used culture techniques or even to embryonic tissue grafts, which have revealed many key mechanisms of development $[12,13]$, makes it possible to assess differentiation at the single-cell level. Epithelial cells can be mixed with mesenchymal cells, endothelial cells or any type of cell one can imagine. Small molecules, recombinant proteins or viruses can be used to modulate and test the outcome in organoid cultures. Organoid culture techniques for lung cells have recently been reviewed [14], but it is important to highlight some key aspects of these types of study.

Three-dimensional co-culture and co-transplantation organoid systems have begun to define the cell-cell crosstalk between epithelial progenitors, endothelial cells and mesenchymal cells in the lung. A fascinating finding culminating from numerous studies over many years has shown that distal lung epithelial cells require stromal cell inputs (perhaps secretory and structural in nature) to thrive in culture, whereas cultured tracheal epithelial cells do not require stroma $[1,2,5,6,15,16]$. In this way, tracheal basal cells resemble the famous intestinal stem cells marked by Lgr5 gene expression. Single $\operatorname{Lgr} 5^{+}$cells can form organoids without additional stroma and give rise to structures like intestinal crypts in culture, and $\mathrm{Ngfr}{ }^{+}$ tracheal cells make tracheospheres containing secretory airway cell types without accessory cell types [5]. Although stromal cells are not needed to derive tracheospheres per se, in vivo tracheal cells have critical interactions with other cell types that are an important aspect of their regulation. Adding various stromal cells to tracheosphere assays may therefore offer an opportunity to understand the relevant cell-cell signalling that occurs in vivo. Distal epithelial progenitor cells have been nurtured in combination with mouse embryonic fibroblasts, immortalised and primary lung fibroblasts (e.g. MLg cells, Pdgfr-expressing cells) $[1,2,6,15]$. We created an organoid co-culture system that revealed unique mechanisms by which endothelial cells influence differentiation and repair in lung epithelium [16]. Importantly, we were able to derive multi-lineage organoids even from single lung progenitor cells in these three-dimensional co-cultures, demonstrating that individual distal lung epithelial cells can give rise to bronchiolar and alveolar cell types (figure 1). In our studies, we have been able to passage organoids at least seven times in culture, demonstrating that long-term cultures are possible with distal lung epithelia. As a field, we can now visualise the formation of airway- and alveolar-like structures from single cells, and we have a way to precisely define how differentiation is controlled at the molecular level. For example, we showed that lung epithelial progenitors and lung endothelial cells interact in a novel BMP4-NFATc1-thrombospondin-1 signalling axis that drives lineage-specific differentiation [16]. We have recently used our organoid system to define lung mesenchymal cell types that specifically regulate airway or alveolar epithelial cells (unpublished data). It is now possible with the systems available to assess the function of diverse lung epithelial progenitor cells and their mesenchymal and endothelial partners.

Until now, systems have not been available to understand which, if any, lung diseases are a result of failure of lung progenitor cell activity or altered cell-cell interactions between epithelial progenitors and their regulatory stromal cells. The tools developed by others as well as by ourselves now make it possible to understand the cell-autonomous and paracrine mechanisms of lung progenitor cell biology that may be 

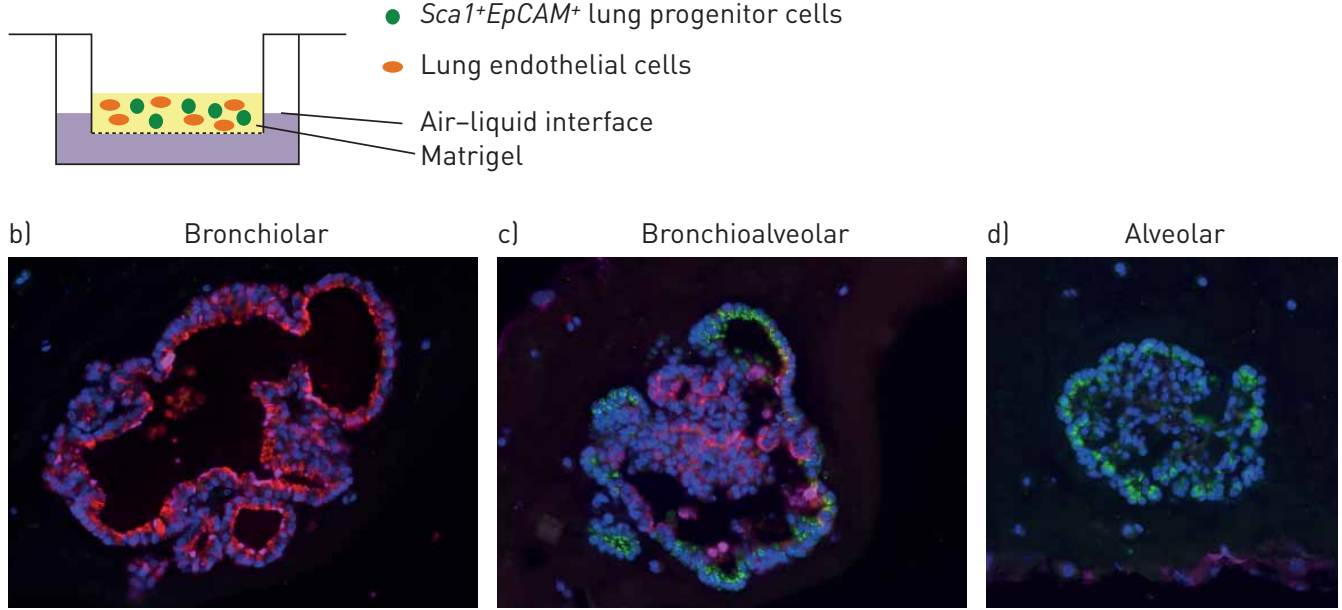

FIGURE 1 Lung progenitor co-cultures yield lung epithelial organoids. a) Schematic of organoid co-culture system. Green represents the lung epithelial progenitor cells that give rise to organoids: $G F P^{+} S c a 1^{+} E p C A M^{+}$ cells isolated by fluorescence-activated cell sorting from $\beta$-actin-GFP mice are shown. Orange represents lung endothelial cells used in the co-culture to provide support to epithelial cells. Yellow represents Matrigel extracellular matrix mixture. Cells suspended in Matrigel in a transwell are submerged partially in the medium to achieve an air-liquid interface culture. b-d) Representative images of the three types of organoid that can be derived from Sca1 $1^{+} \mathrm{CAM}^{+}$lung epithelial progenitor cells in co-cultures with endothelial cells. b) Representative bronchiolar organoid containing cells positive for club cell secretory protein (CCSP) or bronchiolar cell markers; c) bronchioalveolar organoid containing bronchiolar cells and alveolar cells; d) alveolar organoid containing cells positive for surfactant protein C (SPC). All images were obtained from fluorescence imaging by co-staining for CCSP (red) and SPC (green), with 4',6-diamidino-2-phenylindole (DAPI; blue). Images provided courtesy of Samuel P. Rowbotham (Boston Children's Hospital, Boston, MA, USA; Harvard Medical School, Boston, MA, USA; and Harvard Stem Cell Institute, Cambridge, MA, USA).

altered in pulmonary disease. For example, we are exploring how BMP4-NFATc1-thrombospondin-1 signalling affects alveolar differentiation in mouse models of pulmonary fibrosis and the premature infant lung disease bronchopulmonary dysplasia. Seminal studies have established that mutations in surfactant protein $\mathrm{C}$ associated with susceptibility to pulmonary fibrosis affect lung epithelial cells, and have suggested that fibrosis is a disease of progenitor cell failure [17, 18]. New studies have shown that human alveolar lung progenitor cells are impaired in patients with pulmonary fibrosis: organoid cultures were used to show that one signalling pathway regulating innate immune receptors is responsible for one critical defect in fibrosis and that the progenitor cell nature of pulmonary fibrosis can be dissected with organoid techniques [19]. One can imagine that further delineation of the factors that can stimulate progenitor cell proliferation and differentiation, some of which originate from stromal cells, could be beneficial in the therapeutic sense for lung diseases such as idiopathic pulmonary fibrosis that fit the phenotype of "progenitor cell disease." Chronic obstructive pulmonary disease is yet another lung disease that may be a failure of epithelial progenitors or their microenvironment that can be dissected with organoid techniques. An expanded understanding of human lung progenitor cells is a much-needed next step for bridging these concepts for lung disease, but until human lung progenitor cell biology is as advanced as our knowledge of the mouse, organoid systems offer the opportunity to examine the cell-cell crosstalk that is probably altered in lung disease. By using three-dimensional co-cultures, such as that shown in figure 1, one can now determine if endothelial cells from a lung disease model can alter epithelial cell differentiation or if only cell-autonomous mechanisms are at play in lung disease models. Endothelial cells can be substituted with mesenchymal cells to examine epithelial cell-fibroblast interactions, or all three cell types can be combined to achieve an even better model of the cell-cell signalling that occurs in vivo.

\section{In vivo lineage tracing}

In vivo approaches to examine the ability of various progenitor cells to respond to lung injury, via proliferation and differentiation, have also developed significantly in the lung field. The group of Brigid Hogan has published a widely useful Scgb1a1-CreER allele (Scgb1a1 encodes the club cell secretory protein (CCSP), a well-known marker of bronchiolar epithelial cells), enabling lineage tracing of the bronchiolar lineage in a temporal fashion [20]. This work revealed that CCSP-expressing cells self-renew and give rise to ciliated cells, demonstrating their progenitor cell activity. In steady-state homeostasis, this lineage appears to be restricted from contributing to the alveolar epithelial cell pool: use of hyperoxia causing 
severe epithelial damage and probably damage of numerous stromal cells showed that the CCSP lineage remained unable to contribute to alveolar epithelia. In contrast, we and others examined the in vivo capacity of CCSP-expressing lung progenitors using the Scgb1a1-CreER allele and bleomycin to induce alveolar cell injury, which demonstrated the potential of lung epithelial cells expressing the bronchiolar cell marker CCSP to mediate alveolar cell repair in vivo $[21,22]$. These results and other results using tamoxifen-dependent Cre lineage tracing collectively affected the lung field by showing that the cell types contributing to repair in the adult lung differ depending on injury context and can be distinct from the cells that maintain homeostasis [23-25]. Since these studies, state-of-the-art methods using single-cell lineage tracing [26], multicolour clonality reporters [27] and, more recently, new methods to examine histopathological specimens are emerging as ways to clarify how repair occurs in the murine lung. The current challenge is to reach an equivalent understanding of human lung progenitor cell capacity. Until that is achieved, murine organoid culture techniques and the use of sorted cell populations offer an invaluable tool to model differentiation and disease in the dish, and to identify possible pathways for therapeutic intervention. Furthermore, advances made in transplanting lung progenitor cells into injured mice $[7,24]$ set the stage for the much needed development of an in vivo transplantation system to test lung progenitor cells. We envision that organoids derived from co-culture techniques may offer a way to develop transplantation assays for the lung field that will enable further progenitor cell studies and ultimately, one day, may allow the development of cell therapy protocols.

\section{Cancer and stem cells}

Key advances in the biology of lung cancer have also been possible using stem cell approaches. The field of cancer stem cells began with the identification of leukaemia-initiating cells, and the first reports of stem-like cells in solid tumours emerged nearly a decade later. At that time it was unknown if other types of tumour were driven by progenitor-like cells and, if so, what possible vulnerabilities cancer stem cells could reveal as new therapeutic targets. Much as for normal lung progenitor cells, methods to test the ability of lung tumour cells to act as cancer stem cells in vivo needed to be developed. We used genetically engineered mouse models that accurately represent human lung cancer and created an orthotopic transplantation assay to successfully propagate tumours in recipient mice. Using this assay, we identified cancer stem cell populations in the two most common types of lung cancer (figure 2) [28, 29], and we showed that cancer stem cells contribute to metastatic lung cancer [30]. Cultures comparing tumour cell

\section{a) Organoid system}
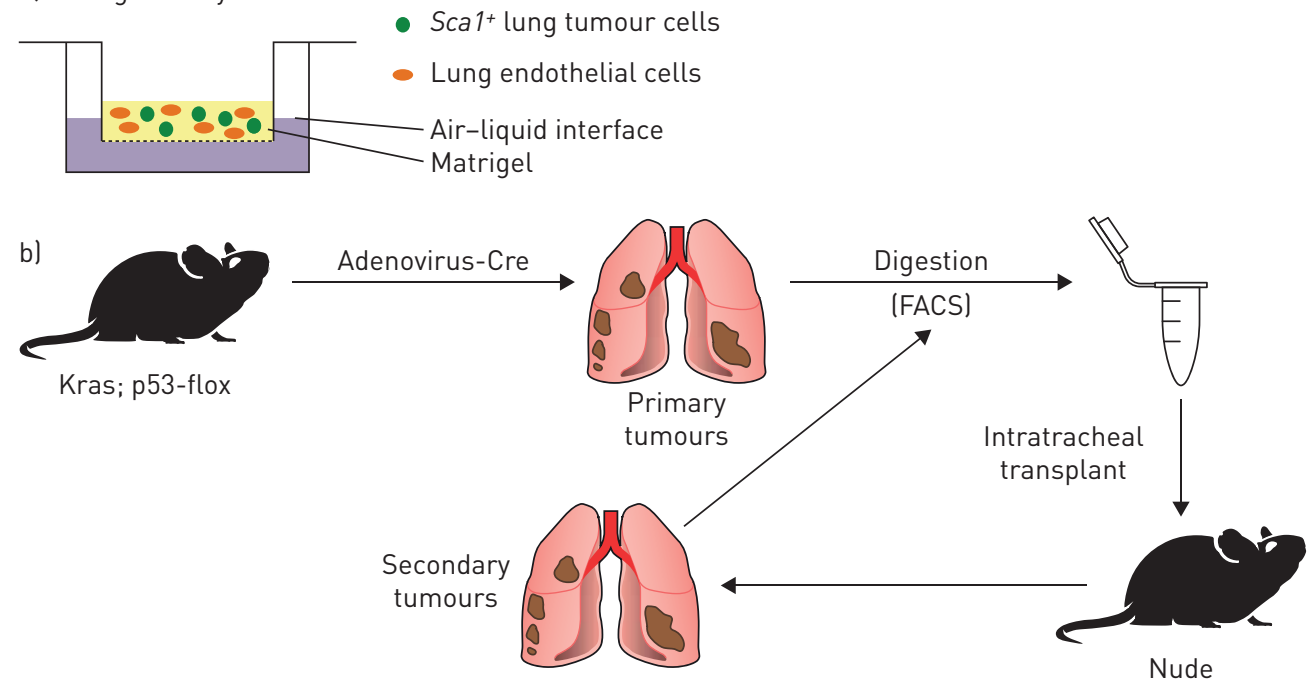

FIGURE 2 Lung tumour-propagating cells can be used in organoid cultures and in vivo transplantation. a) Schematic of organoid co-culture system for tumour cells. Colours are as shown in figure 1a, except that tumour cell subsets (e.g. Sca $1^{+}$tumour cells) are placed in co-culture instead of normal lung progenitors. b) Schematic of assay used to establish tumour-propagating cell activity in vivo. The Lox-stop-lox-Kras-G12D; p53-flox/flox mouse strain is infected intranasally with Adenovirus-Cre to initiate lung tumorigenesis. Lung adenocarcinomas arise and are dissected out for digestion to obtain single-cell suspensions. Tumour cell subpopulations are isolated by fluorescence-activated cell sorting (FACS) and transplanted by intratracheal delivery into the immunosuppressed nude mouse strain. Resulting tumours (referred to as secondary tumours) in the recipient nude mice are indicative of tumour-propagating cell function. Secondary tumours can be dissected and processed for single-cell suspensions in a reiterative process. Reproduced and modified from [28] with permission. 
subpopulations for their ability to give rise to organoids recapitulated in vivo transplantation results: the tumour-propagating cells that are most efficient at organoid formation are the bona fide propagating cells in vivo [29]. Similarly, three-dimensional culture techniques were used in combination with in vivo transplantation to show that CD24 and Notch signalling receptors are markers of cancer stem cells in lung cancer [31]. Numerous studies have reported the identification of tumour-initiating cells in human lung cancer [32]; however, the majority of other studies of this concept used only cell lines, rather than primary tumour cells, to examine progenitor cell activity. Therefore, once again, there is a need to dig deeper into the human equivalent of cancer stem cells to fully understand lung cancer, and, in the absence of a precise definition of human lung cancer progenitors, organoid techniques give us a way to model the tumour microenvironment from accurate murine models and even patient samples. Whereas organoids can be used to model progenitor-like cells in established tumours as described here, this system also offers the opportunity to compare the ability of different lung cell types to act as cells of origin of the various types of lung cancer. As an example, we recently compared the ability of proximal and distal lung progenitors to form organoids after activation of oncogenic Kras or loss of the tumour suppressor $L k b 1$, allowing us to pinpoint the possible cell types that can produce adenosquamous lung cancer in the mouse [33].

\section{Epigenetic regulation}

One clear intersection between normal lung progenitor cells and lung cancer is their shared epigenetic mechanisms. Our work in epigenetics led us to experiments that revealed a new combination therapy approach for particular subsets of lung cancer patients [34-36]. Our most recent studies have aimed to identify the pathways upon which lung cancer stem cells rely, with a focus on epigenetic regulators. Our studies have revealed that an epigenetic enzyme thought to have oncogenic activity in many cancers actually plays a role as a negative regulator of tumour progression and metastasis (unpublished data). Our findings to date suggest that this occurs by the regulation of differentiation mechanisms, as revealed using our normal lung progenitor cell organoid culture techniques. Thus, using lung cancer stem cells and organoid approaches has led to a new understanding of the biology of lung cancer. Importantly, our work also shows how careful we as a field need to be in targeting epigenetic regulators in lung cancer. Whereas epigenetic regulators offer therapeutic opportunity, caution should be taken to determine precise therapeutic windows (e.g. at different stages of tumorigenesis) and how the most aggressive lung cancer cells respond to therapy. Modelling these responses with stem cell techniques can pinpoint vulnerabilities and dangers.

\section{Summary}

Current and future work will build on these discoveries and tools, to lead the field towards a better understanding of stem cell biology in the lung, the development of innovative approaches for examining the cellular and molecular basis of lung disease and cancer, and identification of new therapeutic avenues. Regardless of the remaining questions about the exact identity and nature of progenitor cells in human and even murine lung, there is no question that stem cell approaches have made new connections regarding how lung disease and lung cancer are driven. Our challenge as a field is now to utilise these systems to the highest level of our imagination to map the vulnerabilities of disease.

\section{References}

1 Kim CFB, Jackson EL, Woolfenden AE, et al. Identification of bronchioalveolar stem cells in normal lung and lung cancer. Cell 2005; 121: 823-835.

2 McQualter JL, Yuen K, Williams B, et al. Evidence of an epithelial stem/progenitor cell hierarchy in the adult mouse lung. Proc Natl Acad Sci USA 2010; 107: 1414-1419.

3 Teisanu RM, Lagasse E, Whitesides JF, et al. Prospective isolation of bronchiolar stem cells based upon immunophenotypic and autofluorescence characteristics. Stem Cells 2009; 27: 612-622.

4 Teisanu RM, Chen H, Matsumoto K, et al. Functional analysis of two distinct bronchiolar progenitors during lung injury and repair. Am J Respir Cell Mol Biol 2011; 44: 794-803.

5 Rock JR, Onaitis MW, Rawlins EL, et al. Basal cells as stem cells of the mouse trachea and human airway epithelium. Proc Natl Acad Sci USA 2009; 106: 12771-12775.

6 Chen H, Matsumoto K, Brockway BL, et al. Airway epithelial progenitors are region specific and show differential responses to bleomycin-induced lung injury. Stem Cells 2012; 30: 1948-1960.

7 Chapman HA, Li X, Alexander JP, et al. Integrin $\alpha 6 \beta 4$ identifies an adult distal lung epithelial population with regenerative potential in mice. J Clin Invest 2011; 121: 2855-2862.

8 You Y, Brody SL. Culture and differentiation of mouse tracheal epithelial cells. Methods Mol Biol 2013; 945 : 123-143.

9 Dobbs LG. Isolation and culture of alveolar type II cells. Am J Physiol 1990; 258: L134-L147.

10 Beers MF, Moodley Y, RCMB Stem Cell Working group. When is an alveolar type 2 cell an alveolar type 2 cell? A conundrum for lung stem cell biology and regenerative medicine. Am J Respir Cell Mol Biol 2017; in press [https:// doi.org/10.1165/rcmb.2016-0426PS].

11 Simian M, Bissell MJ. Organoids: a historical perspective of thinking in three dimensions. J Cell Biol 2017; 216: $31-40$. 
12 Shannon JM. Induction of alveolar type II cell differentiation in fetal tracheal epithelium by grafted distal lung mesenchyme. Dev Biol 1994; 166: 600-614.

13 Shannon JM, Nielsen LD, Gebb SA, et al. Mesenchyme specifies epithelial differentiation in reciprocal recombinants of embryonic lung and trachea. Dev Dyn 1998; 212: 482-494.

14 Barkauskas CE, Chung MI, Fioret B, et al. Lung organoids: current uses and future promise. Development 2017; 144: 986-997.

15 Barkauskas CE, Cronce MJ, Rackley CR, et al. Type 2 alveolar cells are stem cells in adult lung. J Clin Invest 2013; 123: 3025-3036.

16 Lee JH, Bhang $\mathrm{DH}$, Beede $\mathrm{A}$, et al. Lung stem cell differentiation in mice directed by endothelial cells via a BMP4-NFATc1-thrombospondin-1 axis. Cell 2014; 156: 440-455.

17 Blackwell TS, Tager AM, Borok Z, et al. Future directions in idiopathic pulmonary fibrosis research. An NHLBI workshop report. Am J Respir Crit Care Med 2014; 189: 214-222.

18 Mulugeta S, Nureki S, Beers MF. Lost after translation: insights from pulmonary surfactant for understanding the role of alveolar epithelial dysfunction and cellular quality control in fibrotic lung disease. Am J Physiol Lung Cell Mol Physiol 2015; 309: L507-L525.

19 Liang J, Zhang Y, Xie T, et al. Hyaluronan and TLR4 promote surfactant-protein-C-positive alveolar progenitor cell renewal and prevent severe pulmonary fibrosis in mice. Nat Med 2016; 22: 1285-1293.

20 Rawlins EL, Okubo T, Xue Y, et al. The role of Scgbla1 ${ }^{+}$Clara cells in the long-term maintenance and repair of lung airway, but not alveolar, epithelium. Cell Stem Cell 2009; 4: 525-534.

21 Tropea K, Leder E, Aslam M, et al. Bronchioalveolar stem cells increase after mesenchymal stromal cell treatment in a mouse model of bronchopulmonary dysplasia. Am J Physiol Lung Cell Mol Physiol 2012; 302: L829-L837.

22 Rock JR, Barkauskas CE, Cronce MJ, et al. Multiple stromal populations contribute to pulmonary fibrosis without evidence for epithelial to mesenchymal transition. Proc Natl Acad Sci USA 2011; 108: E1475-E1483.

23 Giangreco A, Arwert EN, Rosewell IR, et al. Stem cells are dispensable for lung homeostasis but restore airways after injury. Proc Natl Acad Sci USA 2009; 106: 9286-9291.

24 Vaughan AE, Brumwell AN, Xi Y, et al. Lineage-negative progenitors mobilize to regenerate lung epithelium after major injury. Nature 2015; 517: 621-625.

25 Desai TJ, Brownfield DG, Krasnow MA. Alveolar progenitor and stem cells in lung development, renewal and cancer. Nature 2014; 507: 190-194.

26 Kumar ME, Bogard PE, Espinoza FH, et al. Mesenchymal cells. Defining a mesenchymal progenitor niche at single-cell resolution. Science 2014; 346: 1258810.

27 McConnell AM, Yao C, Yeckes AR, et al. p53 regulates progenitor cell quiescence and differentiation in the airway. Cell Rep 2016; 17: 2173-2182.

28 Curtis SJ, Sinkevicius KW, Li D, et al. Primary tumor genotype is an important determinant in identification of lung cancer propagating cells. Cell Stem Cell 2010; 7: 127-133.

$29 \mathrm{Xu}$ C, Fillmore CM, Koyama S, et al. Loss of Lkb1 and Pten leads to lung squamous cell carcinoma with elevated PD-L1 expression. Cancer Cell 2014; 25: 590-604.

30 Lau AN, Curtis SJ, Fillmore CM, et al. Tumor-propagating cells and Yap/Taz activity contribute to lung tumor progression and metastasis. EMBO J 2014; 33: 468-481.

31 Zheng Y, de la Cruz CC, Sayles LC, et al. A rare population of $\mathrm{CD} 24^{+} \mathrm{ITGB}^{+} \mathrm{Notch}^{\mathrm{hi}}$ cells drives tumor propagation in NSCLC and requires Notch3 for self-renewal. Cancer Cell 2013; 24: 59-74.

32 Zhang WC, Shyh-Chang N, Yang $\mathrm{H}$, et al. Glycine decarboxylase activity drives non-small cell lung cancer tumor-initiating cells and tumorigenesis. Cell 2012; 148: 259-272.

33 Zhang H, Fillmore Brainson C, Koyama S, et al. Lkb1 inactivation drives lung cancer lineage switching governed by Polycomb Repressive Complex 2. Nat Commun 2017; 8: 14922.

34 Dovey J, Zacharek S, Kim CF, et al. Bmil is critical for lung tumorigenesis and bronchioalveolar stem cell expansion. Proc Natl Acad Sci USA 2008; 105: 11857-11862.

35 Zacharek SJ, Fillmore CF, Lau AN, et al. Lung stem cell self-renewal relies on Bmil-dependent control of expression at imprinted loci. Cell Stem Cell 2011; 9: 272-281.

36 Fillmore CM, Xu C, Desai PT, et al. EZH2 inhibition sensitizes BRG1 and EGFR mutant lung tumors to TopoII inhibitors. Nature 2015; 520: 239-242. 